\title{
Robust, precise and scalable: A phase-encoded pattern for visual $X, Y, \Theta$ positioning*
}

\author{
Antoine N. André ${ }^{\text {, Patrick Sandoz }}{ }^{1}$, Maxime Jacquot ${ }^{1}$ and Guillaume J. Laurent ${ }^{1}$
}

\begin{abstract}
At small scales, vision is a widely used contact-less sensor allowing multi-dimensional measurements, notably position, orientation, displacements and relative distances. The most convenient procedure consists in processing natural features present in images of the scene of interest. However, this approach results in performances that are object-dependent, sensitive to noise and restricted to measurement ranges smaller than the field of observation. Different kinds of structured patterns attached to the moving object of interest were proposed to overcome these limitations. This paper presents a phase-based visual $X, Y, \Theta$ positioning method combining nanometric resolution, large measurement range and robustness against common types of images alterations. The paper describes the phase-based position coding/decoding principles and then demonstrates the large range-to-resolution ratio as well as the method capabilities to reconstruct the correct position despite significant levels of image noise/occlusion. Finally, the trade-off between image size, resolution obtained and computation speed is discussed.
\end{abstract}

\section{Index Terms}

Computer-vision, Large range-to-resolution ratio, Phase-based measurements, Absolute positioning, nano-positioning

\section{INTRODUCTION}

Automation and manipulation at small scales implies inescapable requirements, notably in regard to the spatial footprint of sensors and actuators and to the nature and impact of contacts. In this context, computer vision provides a convenient tool for the simultaneous control of diverse parameters, among which position, displacement, orientation and relative distances are necessary to most of applications. Computer vision has been widely used for pose/displacement estimation and the reported methods can be distinguished between two categories. The first one exploits the image features naturally present in the scene of interest with either local or global approaches [1], [2], [3]. Local methods rely on the detection of edges and diverse kinds of gradient analysis whereas global methods are related to digital image correlation principles [4], [5], [6]. The ability to retrieve pose/displacement from features naturally present in the scene is an indisputable strength of those methods. Unfortunately, the associated drawback is that performances are highly image-dependent and sensitive to the signal-to-noise ratio. The second category of methods relies on the insertion of diverse kinds of structured patterns in the scene of interest in order to secure a sufficient amount of information relevant to the pose/displacement retrieval algorithms. The higher level of constraints associated to those methods results in improved performances, namely highly subpixelic resolutions, image-independent performances and robustness against noise [7], [8]. Furthermore, thanks to the knowledge of the pattern structure, those methods can be self-calibrating and allow a measurement range that is no longer limited by the vision system field of observation but by the physical extension of the pattern. In this field, the choice of pseudo-periodic patterns associated to Fourier-like phase computations seems to be the right option to obtain best performances, notably in regard to the range-to-resolution ratio.

The $X, Y, \Theta$ positioning method presented in this paper belongs to the second category of approaches. It makes use of a pseudo-periodic pattern combining a periodic frame aimed to ensure high-resolution but relative phase measurements with a complementary binary encryption suited to convert relative displacements into absolute position and orientation. The nominal performances of the method have been presented recently with a pattern period of $9 \mu \mathrm{m}$ and a $10 \times$ microscope objective achieving a resolution below one nanometer over a surface of $10 \times 10$ centimeters [9]. This complementary paper presents the scalable property of the method through experiments carried out with scaled periods from 60 to $1200 \mu \mathrm{m}$ observed with a zoom lens allowing a standoff distance of more than $20 \mathrm{~cm}$ and focuses on the robustness of the method that, thanks to phase-based decoding algorithms, is compliant to high physical period scaling with low precision loss.

\section{Phase-encoded position Reference PATtern}

\section{A. Position encoding}

The pseudo-periodic pattern designed is represented in Fig. 1. It corresponds to a 2D periodic distribution of dots that results from the intersection of vertical lines encoding for the horizontal direction and with horizontal lines encoding for the vertical direction. The periodic frame is aimed to define sharp spatial frequency carriers useful for further narrow band-pass filtering in the spectral domain and efficient noise rejection. The alterations of the periodic frame are necessary to discriminate between consecutive periods and to allow the unambiguous determination of every period order as counted from the top-left corner

\footnotetext{
*This work was supported in part by the Cross-disciplinary Research (EIPHI) Graduate school (ANR-17-EURE-0002), in part by Region Bourgogne Franche-Comt (Nano6D), in part by ROBOTEX (ANR-10-EQPX-44-01) and in part by the I-SITE Bourgogne Franche-Comté project (ANR-15-IDEX-03).

${ }^{1}$ The authors are with the FEMTO-ST Institute, Univ. Bourgogne Franche-Comté, UMR CNRS 6174, 25000 Besançon, France \{antoine.andre, patrick.sandoz, maxime.jacquot, guillaume.laurent\}@femto-st.fr
} 


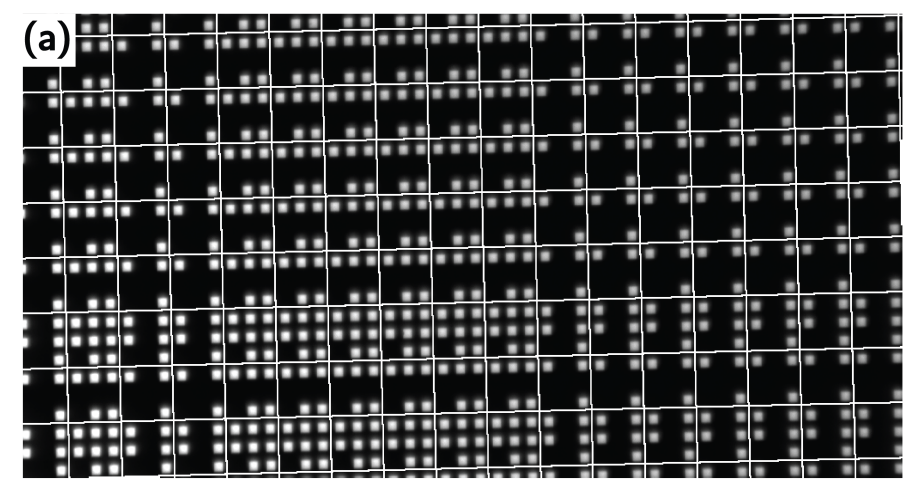

(b)

$$
\left[\begin{array}{ccc}
O F F & x_{\text {bit }} & O N \\
y_{\text {bit }} & x_{\text {bit }} \cdot y_{b i t} & y_{b i t} \\
O N & x_{\text {bit }} & O N
\end{array}\right]
$$

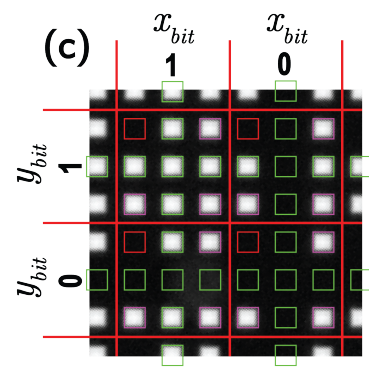

Fig. 1. Pseudo-periodic pattern and coding principle. (a) Experimental image of the pattern. White lines delimit one bit in both $X$ and $Y$ directions as used for absolute position encryption. (b) Rules for every $3 \times 3$ coding cell versus the $X$ and $Y$ bit values. (c) Coding cells obtained for the 4 ( $x, y$ ) binary combinations and for a single angular direction. Colored squares discriminate coding spots from non-coding ones.

of the whole pattern. This binary encoding is realized by sets of three consecutive periods as delimited by the white lines of Fig. 1. The central period of each set of three is turned ON when the associated bit is equal to '1' and turned OFF otherwise. The intersection of bits along both directions results thus in elementary coding cells of $3 \times 3$ dots as depicted in Fig. 1 b,c. Among these sets of 9 dots, two are coding only for the $X$ direction and two others only for the $Y$ direction. The central dot depends on the binary values of both $X$ and $Y$ directions whereas only three corners are turned ON in order to avoid $\pi / 2$ rotation ambiguities. Fig. 11.c presents the four coding cells possibilities for a single angular quadrant.

The period order encryption is based on linear feedback shift register (LFSR) sequences that present the advantage of requiring a single binary track whatever the bit depth ' $n$ ' of the binary word chosen [10]. Then, the position of any local zone of the pattern can be reconstructed provided that, in both directions, at least $3 n$ periods appear in the recorded image. The maximal extension of the pattern is $3 \cdot\left(2^{n}-1\right)$ periods allowing a ratio between the measurement range and the minimal field of observation of $\left(2^{n}-1\right) / n$. This concise description of the encoding principle aims to keep room for experimental results and more details can be found elsewhere [9].

\section{B. Phase-based position decoding}

The position reconstruction consists in retrieving the exact $X, Y, \Theta$ localization of the current image recorded with respect to the whole encoded pattern. This involves two phase-based processing steps, respectively linear and binary. The first linear step aims to retrieve the fine position of the dot distribution with respect to the pixel frame of the camera. This linear step provides the high resolution of the method and relies on classical spectral filtering in the Fourier domain as explained with full details in [11]. This processing results in two unwrapped phase maps, associated to the two directions of the pattern and representative of the position of the current image within the whole pattern modulo unknown phase constants $2 k_{x} \pi$ and $2 k_{y} \pi$. The purpose of the binary step is to determine the correct $2 k_{x} \pi$ and $2 k_{y} \pi$ phase adjustments necessary to remove the uncertainty of an entire number of pattern periods along both directions by identifying the relevant binary words encoded in the missing lines and columns of dots. A specificity of the proposed method is to use the linear phase data in this binary decoding in order to strengthen the position detection robustness.

The phase-reinforcement of the binary detection is depicted in Fig. 2 Thanks to the linear phase analysis, the sites of the dot centers are identified as pixels where the wrapped phases are close to 0 ; for instance where $\left|\phi_{1}(i, j)\right|<\pi / 4$ AND $\left|\phi_{2}(i, j)\right|<\pi / 4$, with $\phi_{1,2}(i, j)$ the wrapped phases at pixel (i,j) for both pattern directions 1 and 2 respectively. Similarly, the image background can be identified as pixels where either $\left|\phi_{1}(i, j)\right|>3 \pi / 4$ OR $\left|\phi_{2}(i, j)\right|>3 \pi / 4$. The application of these phase thresholds to the image represented in Fig. 1 a results in Fig. 2 a and b, leading to the binary dots detection represented in Fig. 2 c. The analysis of this reduced binary image results in Fig. 1 1 c with the dot sites determination. The unwrapped phase 
(a) foreground detection

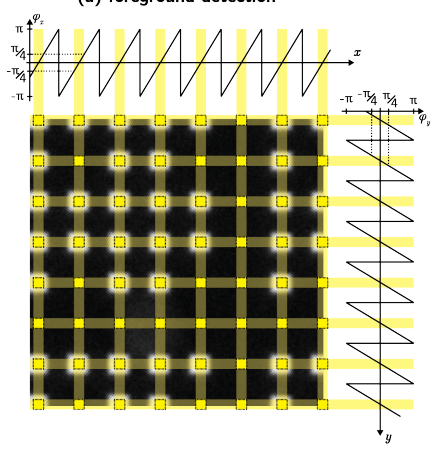

(b) background detection

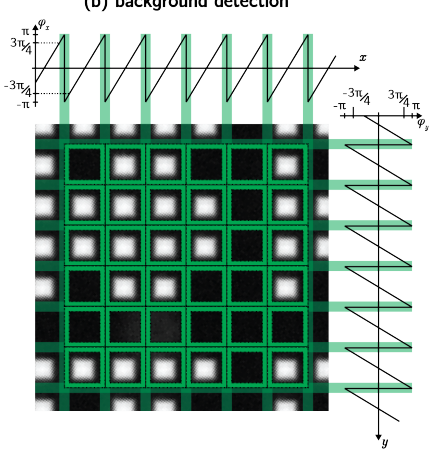

(c) binary image retrieval

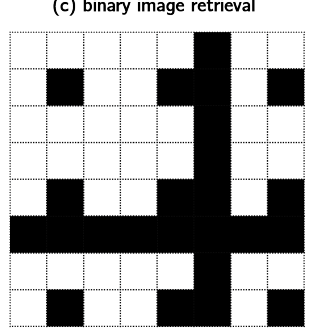

Fig. 2. Phase-based absolute position retrieval. (a), (b) The foreground and background detection is made possible by the image phase analysis. (c) By applying a threshold on the foreground/background selection, a reduced image containing only binary values of the dots can be obtained.

maps are also used to identify pixels belonging to the same lines of dots, respectively columns of dots, as pixels having the same phase constant $k_{1,2}$; i.e. where $2 k_{1,2} \pi-\pi<\Phi_{1,2}(i, j) \leq 2 k_{1,2} \pi+\pi$, with $\Phi_{1,2}(i, j)$ standing for the unwrapped phase in both directions respectively. Then, by comparing the mean intensity at the dot sites modulo three periods, the distribution of the coding cells is retrieved as depicted by the red lines in Fig. 1 $\mathrm{c}$. In this snapshot view, the always missing dots appear in red, the always present dots in magenta whereas dots coding either for the $X$ or $Y$ directions appear in green.

This phase-based discrimination between foreground and background pixels, and between present, absent and coding dots, allows the definition of self-adaptive local decision thresholds for the determination of the binary value of coding lines and columns. In particular, dots located at the center of the coding cells and dependent on the binary value along both directions are discarded from the binary decoding process to avoid cross-talk. This dot by dot value determination leads to the creation of a reduced image containing the binary values of each dot. In addition to the robustness of the method, this binary image saves time processing, therefore allowing more real-time position determination as shown in the next section.

\section{EXPERIMENTAL ANALYSIS}

To validate the method of pattern positioning presented above at different scales, experiments are put in practice. They focus on the range extension capability and on the real time analysis. As this paper demonstrates proofs of feasibility, the encoded patterns used are generated via a smartphone screen. Chen et al. proved that using a smartphone to generate gratings was not a limitation to high resolutions performances [12]. Use of display grids are very handful since they are low-cost and easy to implement on any system. Furthermore, the use of a macro lens allows the measure to be deported at approximately $20 \mathrm{~cm}$ from the pattern, while microscopic objectives have shorter working distances (around $1 \mathrm{~cm}$ ) thus restricting the applications fields to short working distance systems.

\section{A. Experimental setup}

A test of performances comparison between different physical period defining the pattern are made to study the link with achievable resolutions. Ultimate performances described in a previous article [9] will serve as a reference. These performances were measured with an experimental setup involving microscopic objective while presented experiments are made at a more macroscopic scale which can lead to new kind of robotics applications.

The experiments are made in a metrological room (with temperature and humidity regulation) built on a independent basement. The practical setup is placed on an anti-vibration table and is composed by a camera (IDS UI-3280CP-M, set at 20 images per second saved on 12 bits of depth), a macroscopic objective (Computar $10 \times$ ) and a pattern displayed on a smartphone (LG G2). To increase the stability of the experimental setup and reduce the impact of environmental noises, all the above described parts are assembled on the same optical rail as shown on Fig. 3 . 


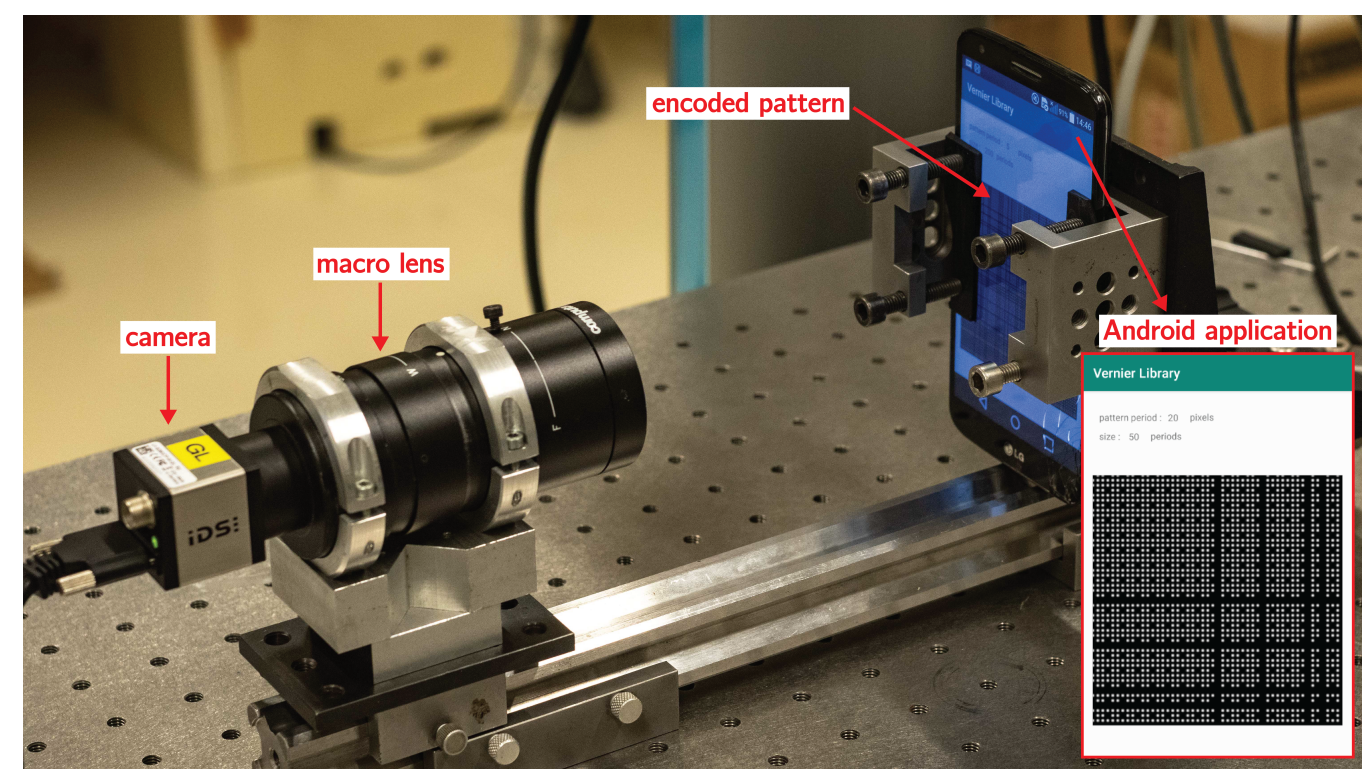

Fig. 3. Experimental setup showing the camera, the objective and the smartphone used for pattern display.

The smartphone application was developed in Android and can generate different sizes of pattern, based on the choice of the pixelic period made by the user. As the smartphone screen can display colors on 8 bits of depth, discrete sinusoidal functions are used for the generation. This allows an almost perfect periodic sinusoidal pattern to be generated; then a mask is applied on the pattern accordingly to the method presented in sec. II-A to generate the coding part of the pattern. With a screen of 424 pixels per inch, the physical pixel size can be determined as $59.29 \mu \mathrm{m}$.

\section{B. Scalable physical period study}

As described in Sec. II-A, the proposed method is applicable to any physical period and with any number of bits for the LFSR coded sequence generation. With the smartphone tool to generate any kind of encoded pattern, a study on the impact of the physical period size on the measured precision is done. The pattern is generated with a 8 bits LFSR sequence and physical periods used go from 1 pixel to 20 pixels (i.e. from $60 \mu \mathrm{m}$ to $1.2 \mathrm{~mm}$ ). Scaling the physical period have the advantage to also scale the global pattern size and can therefore be a precious compliant tool that can fit on numerous kinds of applications. With mentioned used physical periods, generated patterns size from 4.6 to $92 \mathrm{~cm}$.

The experiment is made to study the noise of the measure and performances limits of the method. Results of standard deviation (STD) along the three axis $x, y$ and $\theta$ are shown in Table I The presented $9 \mu \mathrm{m}$ period size is used as a comparison for other physical periods used.

TABLE I

EXPERIMENTAL STD (OUT OF 80 IMAGES) WITH DIFFERENT PHYSICAL PERIOD SIZES.

\begin{tabular}{|c|c|c|c|}
\hline $\begin{array}{c}\text { Period } \\
\text { size }(\boldsymbol{\mu m})\end{array}$ & $\begin{array}{c}\text { STD along } \\
x \text { axis (nm) }\end{array}$ & $\begin{array}{c}\text { STD along } \\
y \text { axis (nm) }\end{array}$ & $\begin{array}{c}\text { STD of } \\
\theta \text { angle }(\boldsymbol{\mu r a d} .)\end{array}$ \\
\hline $9[9]$ & 0.96 & 0.77 & 1.07 \\
\hline 60 & 7.11 & 6.88 & 1.11 \\
\hline 180 & 10.92 & 9.15 & 1.33 \\
\hline 300 & 14.84 & 11.12 & 1.61 \\
\hline 600 & 18.97 & 14.28 & 1.76 \\
\hline 1200 & 22.09 & 18.02 & 1.98 \\
\hline
\end{tabular}

With this experiment, a chart of achievable resolution depending on the physical period used can be deduced. Fig. 4 presents these results of resolution depending on the physical period tested. Each point of traced lines correspond to the obtained standard deviations value presented in Table. I The major difference between $x$ and $y$ results can be explained by a physical 


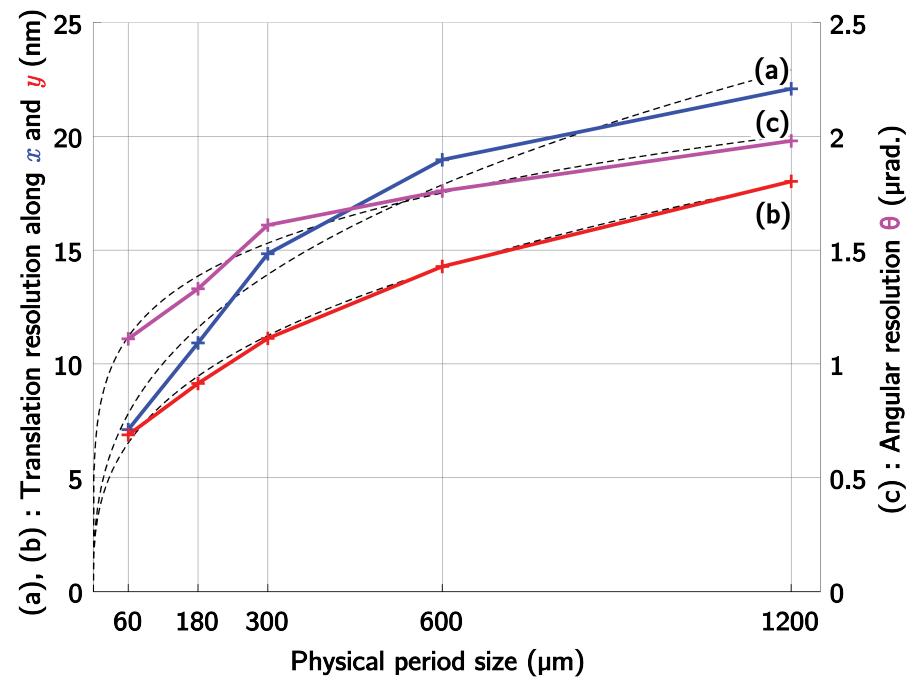

Fig. 4. (a) and (b): achievable resolutions respectively along $x$ and $y$, (c): achievable angular resolution $\theta$ with regard to the physical period used. Dashed lines represent the fitting curves of experimental data.

bias of the imaging sensor which tends to be more accurate along one dimension. To confront these results to a law of pattern design, fitting curves are computed with the following equation:

$$
y=a \cdot x^{b}
$$

where $x$ stands for the targeted physical period, $y$ the corresponding achievable resolution and where $a$ and $b$ are the equation coefficients. The accuracy of the fit is measured with $r^{2}$ values of $97.45 \%$ along the $x$ axis, $99.5 \%$ along the $y$ axis and $97.85 \%$ for the pattern orientation $\theta$.

Even if these results are based on few tested physical period sizes, the highlighted law of resolution prediction based on the physical period becomes a convenient tool for designing a pattern which have to fit precise specifications. Combined with the range scaling capabilities, this law allows a finer determination of a pattern parameters when it comes to practical design and printing (by using a mask generator or a high resolution printer for example).

\section{Image size impact}

For practical implementations such as visual servoing, time processing is a clue to achieve better performances. To study the processing time of the method, an experiment based on window size comparison is made. The same image data set (sizing $1024 \times 1024$ pixels with $180 \mu \mathrm{m}$ period size) is used for as a reference in this study before decreasing the size of the computed images. Fig. 5 shows an acquired pattern image with the different tested windows sizes.

The presented pattern positioning algorithm is implemented in $\mathrm{C}++$ and can be proved to perform with a $O(n \log n)$ complexity (with $n$ the number of pixels contained in the image). This complexity calculation is highly dependant on the complexity of the 2-D Fourier transforms used in the process since the other parts of the program are $O(n)$ computations. By decreasing the size of the computed image, processing time is also affected as shown in Table. II This table also highlights a trade-off choice between time processing and resolution. Since the positioning method is based on frequency analysis, the number of visible periods impacts the resolution and a faster position computing comes at the cost of a lower resolution. However, decreasing the image size and thus the number of visible periods in the image doesn't affect as much translation resolution (by a 1.25 factor for each step of image size reduction) as orientation resolution (which is impacted by a factor 3 for each image size reduction).

Fig. 6 shows that the resolution in translation along the $x$ and $y$ axis is less affected and can conserve high resolution performances despite the low number of periods involved in $256 \times 256$ images. Angular analysis presented in the third graph of Fig. 6also demonstrates that even with smaller images involved, the method can still perform an orientation resolution level of $10 \mu \mathrm{rad}$.

The linear accuracy of the method has been demonstrated to be of $1.25 \%$ in translation and of a few $10-5$ in rotation in [9] with a capacitive sensor used as a reference to measure actual displacements applied. 


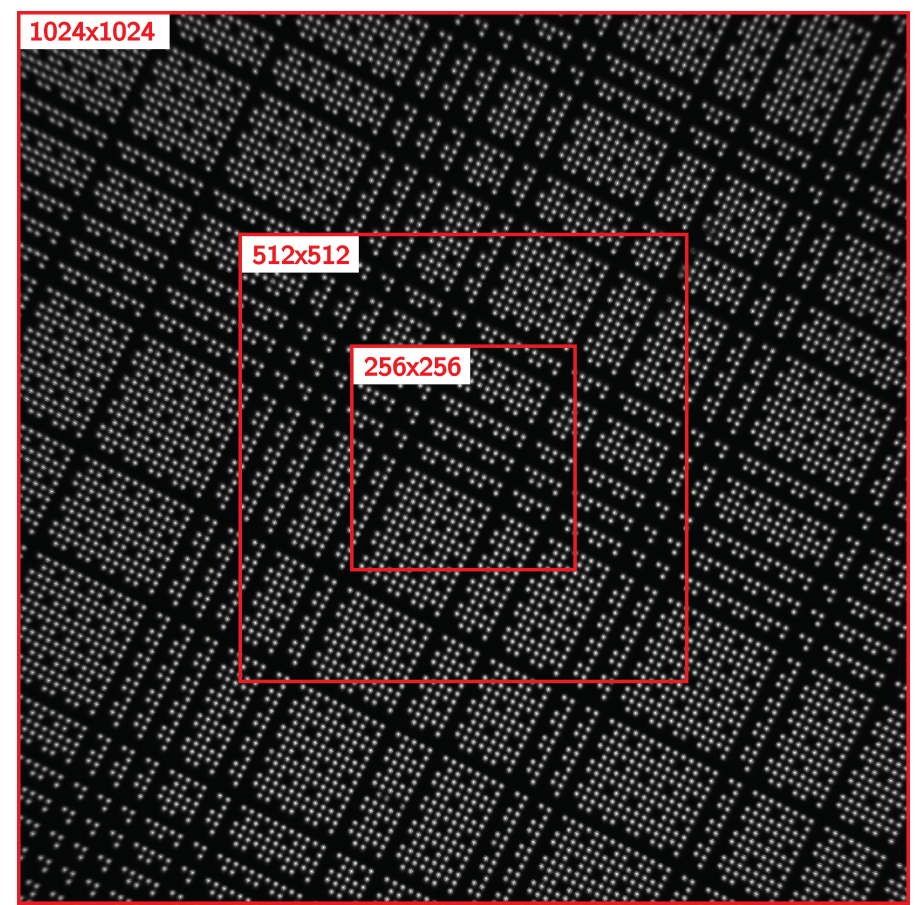

Fig. 5. Different windows size of acquired pattern image used for the trade-off study between image size and achievable resolutions

TABLE II

EXPERIMENTAL STD WITH DIFFERENT WINDOWS SIZE

\begin{tabular}{|c|c|c|c|c|}
\hline $\begin{array}{c}\text { window } \\
\text { size }\end{array}$ & $\begin{array}{c}\text { processing } \\
\text { time }\end{array}$ & $\begin{array}{c}\text { STD along } \\
x \text { axis }\end{array}$ & $\begin{array}{c}\text { STD along } \\
y \text { axis }\end{array}$ & $\begin{array}{c}\text { STD of } \\
\theta \text { angle }\end{array}$ \\
\hline $1024 \times 1024$ & $148.53 \mathrm{~ms}$ & $10.09 \mathrm{~nm}$ & $9.68 \mathrm{~nm}$ & $1.13 \mu \mathrm{rad}$. \\
\hline $512 \times 512$ & $33.07 \mathrm{~ms}$ & $10.76 \mathrm{~nm}$ & $12.14 \mathrm{~nm}$ & $3.28 \mu \mathrm{rad}$. \\
\hline $256 \times 256$ & $7.70 \mathrm{~ms}$ & $14.02 \mathrm{~nm}$ & $14.57 \mathrm{~nm}$ & $10.06 \mu \mathrm{rad}$. \\
\hline
\end{tabular}

\section{Conclusions}

This paper presents a computer-vision method of absolute in-plane pose estimation $(x, y$ and $\theta)$ with nanometric resolution in translation and microradians resolution in orientation and scalable range going from a few centimeters to almost one meter. The presented method uses an LFSR sequence to encode a periodic pattern, leading to the creation of an absolute, $2 \pi$ invariant pattern. To achieve large range high-resolution results, the periodicity of the pattern leading to phase evolution analysis is widely used. The first use of the phase is to determine the high resolution position and second one is to determine the absolute position of the acquired pattern image by searching for the encoded position. By using the phase information to determine the code position of the acquired images, local dot-by-dot threshold is used and therefore is proved to be robust to noise and lighting disparities.

Experiments made with a smartphone screen and a home-made developed application proved the method to be easy and cheap to implement on any system. The physical period scaling and the use of a macro lens (which can be placed with a standoff distance from the screen of about $20 \mathrm{~cm}$ ) can therefore be used to comply with any contactless in-plane pose estimation system.

As the presented method is built on the number of bits generating the coding sequence and on the chosen physical period, studies on resolution evaluation depending on physical period has been conducted. They proved the method to be robust and compliant with scaling effect by determining a law between physical period used and achievable resolutions. Since this method can also be implemented for real-time analysis, experiments on image size effect on the measure have been conducted and proved the method to conserve high resolutions despite using small image sizes which are handful for real-time processing.

Future works that aim for the creation of more detailed encoded periodic patterns design help and data sheet will be carried. GPU implementation for quicker image processing with no resolution loss cost will also be studied. 

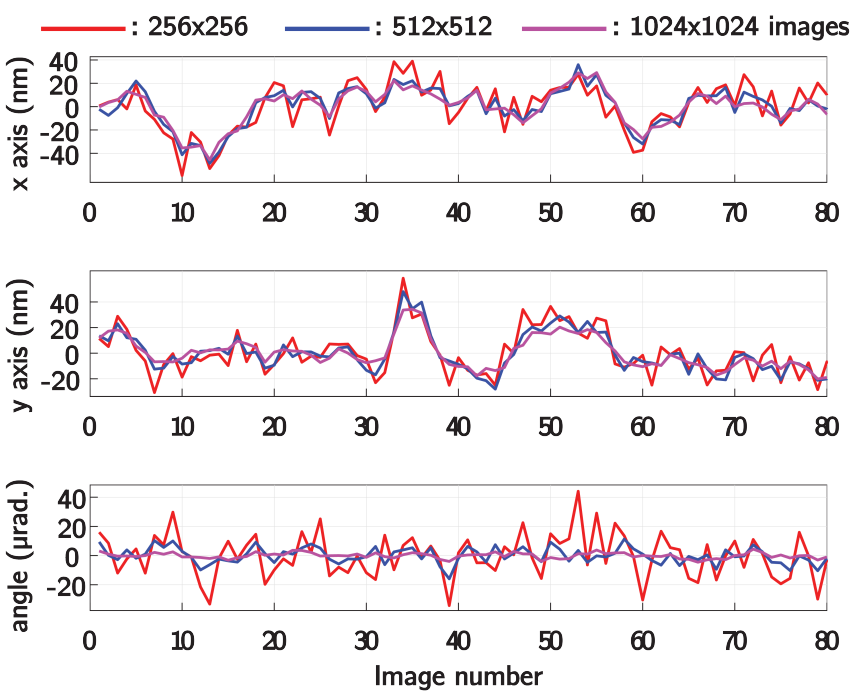

Fig. 6. Static study disparities depending on the image size choice along the three axis $x, y, \theta$

\section{ACKNOWLEDGMENT}

The encoded target has been realized thanks to the RENATECH technological network and its FEMTO-ST facility MIMENTO. Authors acknowledge G. Jutzi, L. Robert, M. Suarez and L. Gauthier-Manuel for technological and experimental assistance.

\section{REFERENCES}

[1] E. Avci, C.-N. Nguyen, K. Ohara, M. Kojima, Y. Mae, and T. Arai, "Towards high-speed automated micromanipulation," in 2013 IEEE Int. Conf. on Robotics and Automation, pp. 1718-1723.

[2] H. K. Chu, J. K. Mills, and W. L. Cleghorn, "Dual-arm micromanipulation and handling of objects through visual images," in 2012 IEEE Int. Conf. on Mechatronics and Automation, pp. 813-818.

[3] J. Liu, Z. Gong, K. Tang, Z. Lu, and Y. Sun, "Locating end-effector tips in automated micromanipulation," in 2013 IEEE Int. Conf. on Robotics and Automation, pp. 1724-1729.

[4] G. Bomarito, J. Hochhalter, T. Ruggles, and A. Cannon, "Increasing accuracy and precision of digital image correlation through pattern optimization," Optics and Lasers in Engineering, vol. 91, pp. 73-85, 2017.

[5] J. Kokorian, F. Buja, and W. M. van Spengen, "In-plane displacement detection with picometer accuracy on a conventional microscope," Journal of Microelectromechanical Systems, vol. 24, no. 3, pp. 618-625, 2014.

[6] H. Li, B. Zhu, Z. Chen, and X. Zhang, "Realtime in-plane displacements tracking of the precision positioning stage based on computer micro-vision," Mechanical Systems and Signal Processing, vol. 124, pp. 111-123, 2019.

[7] H. Sugiura, S. Sakuma, M. Kaneko, and F. Arai, "On-chip method to measure mechanical characteristics of a single cell by using moiré fringe," Micromachines, vol. 6, no. 6, pp. 660-673, 2015.

[8] J.-A. Kim, J. W. Kim, C.-S. Kang, and J. Jin, "Note: An absolute xy- $\theta$ position sensor using a two-dimensional phase-encoded binary scale," Review of Scientific Instruments, vol. 89, no. 4, p. 046105, 2018.

[9] A. N. Andre, P. Sandoz, B. Mauze, M. Jacquot, and G. J. Laurent, "Sensing one nanometer over ten centimeters: A micro-encoded target for visual in-plane position measurement," IEEE/ASME Transactions on Mechatronics, 2020.

[10] J. Stevenson and J. Jordan, "Absolute position measurement using optical detection of coded patterns," Journal of Physics E: Scientific Instruments, vol. 21, no. 12, p. 1140, 1988.

[11] J.-A. Galeano-Zea, P. Sandoz, E. Gaiffe, J.-L. Prétet, and C. Mougin, "Pseudo-periodic encryption of extended 2-d surfaces for high accurate recovery of any random zone by vision," Int. Journal of Optomechatronics, vol. 4, no. 1, pp. 65-82, 2010.

[12] Z.-H. Chen and P. S. Huang, "A vision-based method for planar position measurement," Measurement Science and Technology, vol. 27, no. 12, p. 125018, 2016. 